\title{
Analytic Realization of Relative K-Homology on Manifolds with Boundary and Pairing with K-Cohomology
}

\author{
R. G. Douglas and K. P. Wojciechowski \\ In Honior of the Eightieth Birthday of Professor S. G. Michlin
}

Obwohl K-Homologie abstrakt als Dual der gewöhnlichen K-Theorie von Räumen definiert werden kann, ergibt sich ihre Verwendung in Indextheorie und Analysis durch die konkrete Realisierung, bei der elliptische Operatoren Zyklen sind. Das ist wohlbekannt für Mannig. faltigkeiten ohne Rand, aber nicht für solche mit Rand. In dieser Arbeit geben wir eine Realisierung für relative Zyklen unter Benutzung elliptischër Operatoren auf der verdoppelten Mannigfaltigkeit an und untersuchen Paarungen dieser Zyklen mit K-Kohomologie vermittels Fredholm-Indizes und SpektralfluB.

Хотя K-гомология может быть определена абстрактно как сопряженное к обычнои $K$ теории пространств, ее применение в теории индексов и анализе основывается на конкретном представлении, при котором эллиптические операторы являются циклами. Это хорошо известно для мно́гообразий без края, но не для таких с краем. В цанной работе мы даем реализацию для относительных циклов с использованием эллиптических операторов на удвоенном многообразии и исследуем спаривание этих циклов с К-когомологией посредством индексов Фредгольма и спектрального потока.

Although K-homology can be defined abstractly as a dual of the ordinary K-theory of spaces, its use in index theory and analysis comes about through the concrete realization where elliptic operators are cycles. This is well understood for manifolds without boundary but not. when a boundary is present. In this note we provide a realization for relative cycles using elliptic operators on the doubled manifold and explore pairings of these cycles with $\mathrm{K}$-cohomology in terms of Fredholm indexes and spectral flow.

\section{Introduction}

One consequence of the development of K-homology has been that an index formula can be viewed as the result of the pairing between homology and cohomology in $\mathrm{K}$ theory. Thus détermining the class defined by an elliptic operator. in K-homology becomes paramount and the specificity of an index formula deperids on the concrete, ness of the associated Chern character for $K$-homology. In the case of compact manifolds without boundary there are several good approaches, but we want to mention the one, due to Connes [11], which involves cyclic cohomology and its isomorphism with de Rham homology. Although work continues in this area, one can say with considerable justification that we have a basic understanding of it. This is not case for elliptic operators on manifolds with boundary. There is no systematic theory which incorporates the index theorem 'of Atiyah-Bott [1], the Atiyah-Patodi-Singer theorem [2], and provides a concrete formula for the Chern character of the K-homology class of the operator.

An elliptic operator $A$ on a compact manifold $M$ with boundary can be shown to determine an element [-A] in the Kasparov KK-group-for the algebra $C_{0}(M)$ of func-

1) The research of both authors was partially supported by a grant.from the National Science Foundation. 
tions on $M$ which vanish on $\partial M$ (see [6]). However, determining the image of $[A]$ under the boundary map depends on the calculation of a non-trivial Kasparov product which is not constructive: For that reason BaUm and Douglas [5] offered an alternate definition of the relative group $K_{0}(X, Y)$.in which the boundary map is constructive and in [6] it was shown how to realize $[A]$ in $K_{0}(\dot{M}, \partial M)$.

However, constructing the Chern character for $[A]$ explicitly is more difficult as is describing the connection with the Atiyah-P'atodi-Singer theorem. One way to view the problem is that $C_{0}(M)$ does not have a unit and the tricks introduced by ConNes in [11] for dealing with this do not work. The problem is fundamental. Although one can solve this problem using the one-point compactification, the boundary information is lost and, in particular, the fact that $A$ is smooth up to and including $\partial M$ plays no role. One seeks to define the Chern character on $C(M)$ but there is an obstruction to that which is the image of $[A]$ under the boundary map.

In this note we introduce a different approach to the relative K-homology group $K_{i}(M, \partial M)$ for a manifold with boundary $M$, which is orientable with respect to K-theory. Reflection defines a natural involution $J_{i}$ on the $\mathrm{K}$-theory of the double $X$ of $M$. We show that $K_{i}(M, \partial M)$ is naturally isomorphic to the fixed points of $J_{i}$ on $K_{i}(X)$. This enables one to canonically represent classes in $K_{i}(M ; \partial M)$ by elliptic operators on $X$ with the appropriate behaviour under reflection. One expects to use the well-developed Chern character for operators on $X$ to study the relative K-homology on $M$. Following this approach we obtain explicit calculation of the pairings between relative $K$-homology and $K$-cohomology and the relation of this pairing to the index of concrete operators.

Although part of this has appeared implicitly in the work of others, we believe there is value in considering these results in a systematic fashion.

Formulas involving cyclic homology or de Rham homology, and connections with the Atiyah-Patodi-Singer, theorem will be taken up in a later paper.

\section{Cutting and pasting of elliptic operators}

We recall here a construction from [18], which is crucial for the whole consideration. Let us assume that we have two compact manifolds $M_{1}, M_{2}$ with boundary, such that $\partial M_{1}=Y=\partial M_{2}$ and let us assume that $A_{1}$ and $A_{2}$ are first order elliptic operators defined on $M_{1}$, and $M_{2}$, respectively; $A_{i}: C^{\infty}\left(M_{i} ; E_{i}\right) \rightarrow C^{\infty}\left(M_{i} ; F_{i}\right)$. We assume that they have the following form on some collar' neighbourhood of $Y$ :

$$
A_{\mathrm{i}}=G_{\mathrm{i}}\left(\partial_{\mathrm{i}}+B_{\mathrm{i}}\right) \text {. }
$$

Here $G_{i}(t, \cdot): E_{i}\left|\grave{Y} \rightarrow F_{i}\right| Y$ is a bundle isomorphism, where $t$ denotes the inward normal coordinate and $B_{i}=B_{i}(t, \cdot): C^{\infty}\left(Y^{\prime} ; E_{i} \mid Y\right) \rightarrow C^{\infty}\left(Y ; E_{i} \mid . Y\right)$ is an elliptic differential operator on $Y$. We denote by $a_{i}(x, \xi)$ and $b_{i}(y, \zeta)$ the principal symbols of $A_{i}$ and $B_{i}$, respectively. (We suppress the dependence of $b$ on $t$ to avoid further complicating our notation.) Now we fix Riemannian structures on $M_{i}$ and Hermitian structures on $E_{i}$ and $F_{i}$. If $v$ denotes the unit tangent vector in the $t$ direction, then $a_{i}(t, y ; r \nu+\zeta)=G_{i}(y)\left(i r \cdot I d+b_{i}(y ; \zeta)\right)$ on the collar neighbourhood of $Y$. Now let us assume that there exists a smooth bundle isomorphism $g: E_{\mathrm{l}}\left|Y \rightarrow F_{2}\right| Y$, such that

$$
G_{2}^{*}(y) g(y) b_{1}(y ; \zeta)=b_{2}{ }^{*}(y ; \zeta) G_{2}^{*}(y) g(y)
$$

Under these assumptions we define the manifold $\boldsymbol{X}=M_{1} \dot{\cup} \boldsymbol{M}$ and vector bundles $E^{\sigma}=E_{1} \cup_{0} F_{2}$ and $F^{o}=F_{1} \cup G_{z}^{*} \sigma G_{1}^{-1} E_{2}$ on $X$, where we identify $\dot{e}_{1}$ in $E_{1, y}$ with $g(\dot{y}) e_{1}$ in $F_{2, y}$ to obtain $E^{g}$ and similarly for $F^{0}$. We want to construct an operator on $X$ using $A_{1}^{\prime}$ and $A_{2}$. However, we cannot just glue $A_{1}$ and $A_{2}$ together, because the nor- 


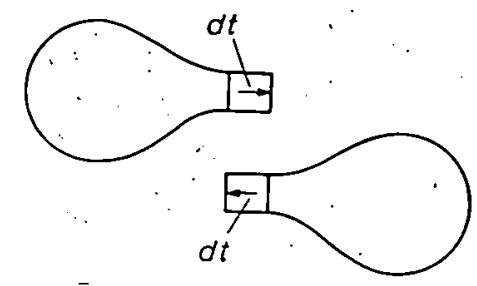

Fig. 1

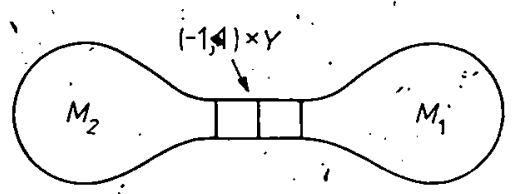

Fig. $\overline{2}$

mal derivatives have opposite orientations (see Fig. 1). To overcome this we use $A_{2}{ }^{*}$ in place of $A_{2}$. Then, we are able to construct an operator which has principal symbol equal to $a_{1}$ on $M_{1}$ and $a_{2}{ }^{\prime}$ ' on $M_{2}$.

We may assume that the intersection of $M_{1}$ and $M_{2}$ is equal to $N=(-1,+1) \times Y$, a bicollar neighbourhood of $Y$ (see Fig: 2 ) and that $t$ is the normal coordinate to $Y$. in $V$. With respect to this coordinate our operators have the following form on $N$ :

$$
A_{1}\left|V=G_{1}\left(\partial_{t}+B_{1}\right), \quad A_{2}^{*}\right| V=G_{2}{ }^{*}\left(\partial_{t}+\left(G_{2}^{*}\right)^{-1} B_{2}{ }^{*} G_{2}{ }^{*}\right)
$$

If for $x=(t, y)$ in $N$; we evaluate $a$ on an element of $E^{g}$. defined by $e$ in $E_{1, x}$, we obtain the element $G_{1}\left(i r v+b_{1}\right) e$ of $F^{\circ}$, which is equal to

$$
\begin{aligned}
\left(G_{2}{ }^{*} g G_{1}{ }^{-1}\right) G_{1}\left(i r+b_{1}\right) e & =\left(i r+b_{2}{ }^{*}\right) G_{2}{ }^{*} g(e) \\
& =G_{2}{ }^{*}\left(i r+\left(G_{2}{ }^{*}\right)^{-1} b_{2}{ }^{*} G_{2}{ }^{*}\right) g(e)=a_{2}{ }^{*}(g(e))
\end{aligned}
$$

This shows that the symbol $a_{1} \cup a_{2}^{*}: \Pi^{*}\left(E^{g}\right) \rightarrow \Pi^{*}\left(F^{g}\right)$ defined by the formula $a_{1} \cup a_{2}^{*}=a_{1}$ on $\Pi^{*}\left(E^{g}\right) \mid M_{1}, a_{1} \cup a_{2}{ }^{*}=a_{2}{ }^{*}$ on $\Pi^{*}\left(E^{0}\right) \mid M_{2}$ is a well-defined elliptic symbol on $X$. We denote by $A_{1} \cup_{g} A_{2}{ }^{*}: C^{\infty}\left(X ; E^{g}\right) \rightarrow C^{\infty}\left(X ; F^{9}\right)$. any elliptic pseudodifferential operator with principal symbol $a_{1} \cup a_{2}^{*}$. In the following we always assume that it is a differential operator on some bicollar neighbourhood of $\boldsymbol{Y}$.

Remark: The operator $A_{1} \cup_{g} A_{2}{ }^{*}$ is only defined up to the $\dot{O}$-order term. We can , define the operator $A_{1} \mathrm{\cup}_{0} A_{2}{ }^{*}$ uniquely. if instead of (1.2) the operator identity $G_{2}^{*} g B_{1}=B_{2}^{*} G_{2} g$ holds.

The most interesting case for us is when $M_{1}=M_{2}=M, A_{1}=A_{2}=A$ and $g=\left(G^{*}\right)^{-1}$. Usually we assume also that $G$ and $B$ do not depend on the nornial coordinate on some suitable collar neighbourhood of the boundary. In this case we simply write $A \cup A^{*}$ for the corresponding operator on $\tilde{M}$, the closed double of $M$.

The preceding construction enables one to "paste together" two elliptic operators defined on two halves of a manifold $X$ under appropriate hypotheses on the operators: Since elliptic operators define the cycles for K-homology, this construction has an interpretation in terms of the Mayer-Viétoris sequence. We will not elaborate on that here.

Next we want to consider an analogous construction for cycles for the ord group in K-homology which involves self-adjoint elliptic operators. We do not treat the most general case. In our discussion of the "odd case" we assume that $A: C^{\infty}\left(M_{;} ; \dot{E}\right)$ $\rightarrow \dot{C}^{\infty}(M ; E)$ is such that

$A \mid V$. is formally self-adjoint,

$$
\because \because \because,\left(A f_{1}, f_{2}\right)=\left(f_{1}, A f_{2}\right) \text { for } f_{1}, f_{2} \in C_{0}^{\infty}(M \backslash Y ; E)
$$

We want to obtain a self-adjoint operator of the form $A \cup( \pm A)$ in $\tilde{M}$. We now assume that $G$ and $B$ do not depend on $t$ on $V$. In that case (1.3) implies $G^{*}=\div G$ and 
$G B=-B^{*} G$. Having in mind the case of generalized Dirac operators we assume further that $G$ is unitary and $B$ is self-adjoint. A section of $E^{G}=E \cup_{G} E$ is a pair $\left(s_{1}, s_{2}\right)$ of sections of $E$ such that $s_{2}=G s_{1}$ on $V$. Since $G A s_{1}=G G\left(\partial_{t}+B\right) s_{1}$ $=-\left(\partial_{t}+B\right) s_{1}$ and $A s_{2}=G\left(\partial_{t}+B\right) s_{2}=+\left(\partial_{t}-B\right) G s_{2}=\left(\partial_{t}+B\right) s_{1}$, it follows that we have a well-defined self-adjoint operator on $\tilde{M}$ of the form $A \cup(-A)$ : $C^{\infty}\left(\tilde{M}, E^{G}\right) \rightarrow C^{\infty}\left(\tilde{M}, E^{G}\right)$

\section{The relative K-homology groups on Spinc-manifolds !}

In this section we discuss an analytic realisation of relative $\mathrm{K}$-homology groups on manifolds with boundary. A related realisation is described in $[5,6]$. Here we show how to represent the relative cycles as the fixed points of the involution defined on the elliptic operators on the closed double of our manifold. This suggests a generalization of our results to more general manifolds which will be discussed elsewhere. We describe in detail the "even" case of the group $K_{0}$ and state the analogous result for $K_{1}$.

Let $M$ be a compact manifold with boundary $\dot{Y}$ and let $\dot{X}$ denote its closed double. We'start by recalling the exact sequence of $C^{*}$-algebras

$$
0 \rightarrow C_{0}(M) \stackrel{j}{\rightarrow} C(X) \stackrel{i}{\rightarrow} C(M) \rightarrow 0
$$

r where $C(X)$, and $C(\dot{M})$ denote the algebras of continuous functions, $C_{0}(M)$ is thè algebra of continuous functions on $M$ which vanish on $Y$, and $j(f)=f \cup 0, k\left(f_{1} \cup f_{2}\right)$ $=f_{2}$. We have also a map $s: C(M) \rightarrow C(X)$ given by the formula $s(f)=f \cup f$, which is a section of $k$, that is, $k s=I d$. The last property implies the long exact sequence in K-theory splits:

$$
0 \rightarrow K^{i}(M, Y) \rightarrow K^{i}(X) \rightarrow K^{i}(M) \rightarrow 0 .
$$

By standard results from K-theory (see [8: Section 19.5]) we obtain a corresponding splitting in K-homology

$$
0 \rightarrow K_{i}(M) \rightarrow K_{i}(X) \rightarrow K_{i}(M, Y) \rightarrow 0 .
$$

Now, let us assume that $M$ is a Spinc-manifold. This induces corresponding Spincstructures on $X$. and $Y$, and hence all these manifolds are K-oriented (see [3]). Thus we have the commutative diagram

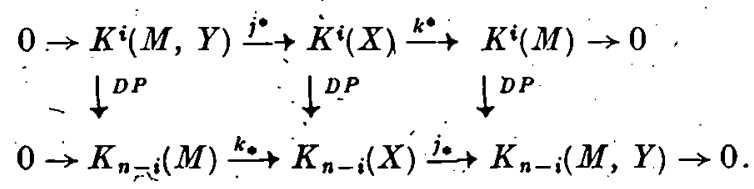

Here $D P$ denotes the Poincaré duality maps which in this case give isomorphisms between corresponding groups and $n=\operatorname{dim} M$. (See [4] for a nice discussion of Poincaré duality in K-homology.) This diagram enables us to present a nice and simple analytic representation of the relative $\mathrm{K}$-homology groups.

We now assume that $M$ is even-dimensional and we deal with the case of even-. dimensional groups. Recall that $K^{0}(M, Y)$ can be identified with the set of triples $(V, W ; h)$, where $V$ and $W$ are vector bundles over $M$ and $h: V\left|Y \rightarrow W_{i}\right| Y$ is a bundle isomorphism. The triple gives the element $\left[V \cup_{n} \cdot W\right]-[W \cup W]$ in $K^{\mathbf{0}}(X)$. This element is "equal to 0 " "on the second copy of $M$ and:the subgroup of elements of this form in $K^{0}(X)$ is, in fact, $K^{0}(M, Y)$. We can see this decomposition from yet 
another point of view: Let $Z=E \cup F$ be a bundle, over $X$. We choose a bundle $\dot{F}^{1}$ on $M$ such that $F \oplus F^{1}=M \times \mathbb{C}^{K}$ and decompose the class of $Z$ in $K(X)$ as follows:

$$
\begin{aligned}
E \cup F & =\left[\left(E \oplus F^{1}\right) \cup\left(F^{\prime} \oplus F^{1}\right)\right]-\left[F^{\perp} \cup F^{1}\right] \\
& =\left[\left(E \oplus F^{1}\right) \cup \mathbb{C}^{K}\right]-\left[F^{\perp} \cup F^{\perp}\right]-[F \cup F]+\left[F \cup F^{\prime}\right] \\
& =\left\{\left[\left(E \oplus F^{1}\right) \cup \mathbb{C}^{K}\right]-\left[\mathbb{C}^{K}\right]\right\}+[F \cup F] .
\end{aligned}
$$

It is clear that the first summand gives us the element of $K^{0}(M, Y)$ and the second the element of $\dot{K^{0}}(M)$.

The next important fact is that on a Spinc-manifold $X$ there exists a natural generator of $K_{0}(X)$ as a $K(X)$-module. This is a class of the Dirac operator $2_{+}: C^{\infty}\left(X ; S^{+}\right)$ $\rightarrow C^{\infty}\left(X, S^{-}\right)$(see $[3,4]$ ). Now $X$ is a manifold of a special kind, since it is a double of $M$, and we can write $X=M \cdot \cup_{Y}(-M)$, where $-M$ denotes $M$ with the reverse orientation. Positive spinors become negative spinors under the change of orientation and it is, in fact, obvious that we can construct a suitable Dirac operator using the Dirac operator on $M$ and the construction from Section 1. Then we have

$$
\hat{\theta}_{X}=\hat{z}_{M} \cup \partial_{M}^{*}: C^{\infty}\left(X ; S^{+} \cup S^{-}\right) \rightarrow C^{\infty}\left(X ; S^{-} \cup S^{+}\right) \text {. }
$$

Now it is easy to describe the element in $K_{0}(X)$ which corresponds to the $(V, W ; h)$ from $K^{0}(M, Y)$. It is the class

$$
\begin{aligned}
& : \quad\left[\left(\hat{\theta} \cup \hat{\boldsymbol{\theta}}^{*}\right) \otimes I d_{V \cup_{n} w}\right]-\left[\left(\hat{\theta} \cup \hat{\partial}^{*}\right) \dot{\otimes} I d_{w \cup w}\right] \\
& ='\left[\left(\partial \cup \partial^{*}\right) \otimes I d_{V U_{B} w}\right]+\left[\left(\partial^{*} \cup \partial\right) \otimes I d_{W \cup W}\right]
\end{aligned}
$$

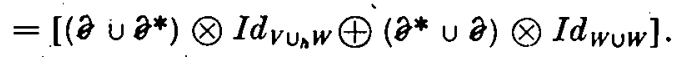

One may ask how to make contact with the group $K_{0}(\dot{M})$. Actually it is not difficult. The point is that the operator

$$
\left(\partial \cup \partial^{*}\right) \otimes I d_{V \cup_{n} W} \oplus\left(\partial^{*} \cup \partial\right) \otimes I d_{W \cup W}
$$

corresponds to the well-defined "local" elliptic boundary value problem. It is well known that the Dirac operator (and $\partial_{M} \otimes I d_{V}$ as well)' does not admit "local" elliptic boundary conditions (see $[2,6,10])$. We define the Balderon projection $P(\dot{\theta})$ as the projection of $C^{\infty}\left(M ; S^{+}\right)$onto the space $H(\dot{\theta})$ of the Cauchy data of $\partial: H(\hat{\theta})=\{u \mid Y$ : $u \in C^{\infty}\left(M ; S^{+}\right), \partial u=0$ in $\left.M \backslash Y\right\}$. One can show that $P(\theta)$ is a pseudodifferential operator of order 0 .

The class of the principal symbol of $P(\hat{\theta})$ in $K^{1}(T Y)$ is an obstruction to the existence $:$, of a" "local" boundary condition. This obstruction vanishes in the case of an operator, which is essentially of the form $\left(\partial^{\oplus} \oplus \partial^{*}\right) \otimes I d$ 'on the collar. The corresponding boundary value problem $R: C^{\infty}\left(X ;\left(S^{+} \otimes V\right) \oplus\left(S^{-} \otimes, W\right)\right) \rightarrow C^{\infty}\left(X ;\left(S^{-} \otimes V\right) \oplus\left(S^{+}\right.\right.$ $\otimes W)) \oplus C^{\infty}\left(Y ;\left(S^{-} \otimes W\right) \mid Y\right)$ is of the form

$$
\begin{aligned}
& \left(s_{1} \otimes v ; s_{2} \otimes w\right) \stackrel{\Re}{\rightarrow}\left(\left(\otimes \otimes I d_{V}\right) s_{1} \otimes v ;\right. \\
& \left.\left(\partial^{*} \otimes I d_{w}\right) s_{2} \otimes w:(G \otimes h) P\left(\xi . \otimes I d_{V}\right) s_{1} \otimes v-P\left(\partial^{*} \otimes I d_{w}\right) s_{2} \otimes w\right)
\end{aligned}
$$

where $s_{1} \otimes v \in \dot{C}^{\infty}\left(X ; S^{+} \otimes V\right), s_{2} \otimes w \in C^{\infty}\left(X ; S^{-} \otimes W\right)$. It is shown in [1] (see [15] for a beautiful exposition) how to deform the symbol of $\mathcal{R}$ to the elliptic symbol on $M$, which is algebraically degenerated at the boundary, i.e. equal to a bundle isomorphism independent of the cotangent vector. on some collar neighbourhood of $Y$. This symbol gives us an element of the group $K^{0}(D M, S M \cup(D M \mid Y))$. We use Poincaré duality once again and obtain $K^{0}(D M, S M \cup D M \mid Y)=K_{0}(D M)=K_{0}(M)$. 
Thus elements of $K_{0}(M)$ are stable homotopy classes of operators which are algebraically degenerated at the boundary or, equivalently are operators, with a "local"" elliptic boundary condition. Let us observe that operator (2.1) has a symbol which is algebraically degenerated on the second copy of $M$, and thus-also on some neighbourhood of the boundary. Hence no deformation is necessary in this case.

We know that an element of $K_{0}(X)$ is given by the class of an operator of the form $\left(\partial \cup \partial^{*}\right) \otimes I d_{E \cup F}$. We decompose this class exactly in the same way as the corresponding class in $K^{0}(X)$ :

$\left(\partial \cup \partial^{*}\right) \cup I d_{E \cup F}=\left\{\left(\hat{\partial} \cup \partial^{*}\right) \otimes I d_{E \oplus \dot{F}^{\perp} \cup \mathbb{C}^{k}} \oplus\left(\hat{\theta}^{*} \cup \hat{\theta}\right) \otimes I d_{\mathbb{C}^{k}}\right\} \oplus\left(\hat{\theta} \cup \hat{\theta}^{*}\right) \otimes I d_{F \cup F}$

The first summand gives us an element of $K_{0}(M)$, and the second represents an element of $K_{0}(M, Y)$.. The essential point in the decomposition is that any cycle here represents the class of a concrete differential operator. Let us consider the class of $A=A_{1} \cup A_{2}$, where $A$ is a first order differential elliptic operator. Thanks to the construction from Section 1 we have the following identities which hold in $K_{0}(X)$ : :

$$
\begin{aligned}
{\left[A_{1} \cup A_{2}\right] } & =\left[A_{1} \cup \dot{A}_{2} \oplus A_{2} \cup A_{2}{ }^{*} \oplus A_{2}{ }^{*} \cup A_{2}\right] \\
& =\left[\left(A_{1} \oplus A_{2}\right) \cup\left(A_{2} \oplus A_{2}{ }^{*}\right)\right]+\left[A_{2}{ }^{*} \cup A_{2}\right] .
\end{aligned}
$$

Once again, it is obvious that the symbol of $A_{2} \oplus A_{2}{ }^{*}$ can be deformed to a symbol of the form $|\xi| I d$ outside of a certain collar neighbourhood of $Y$.-Thus we can deform the operator $\left(A_{1} \oplus A_{2}\right) \cup\left(A_{2} \oplus A_{2}{ }^{*}\right)$ through elliptic pseudodifferential operators to an operator of the form $(C \cup I \dot{d}) \sqrt{\Delta}$, where $C \cup I d$ is an elliptic pseudodifferential operator of order 0 and $\sqrt{\Delta}$ is the square root of a suitable Laplacian. The class of this operator $C \cup I d$ belongs to $K_{0}(M)$ and the class of $A_{2}{ }^{*} \cup A_{2}$ gives an element of $K_{0}(M, Y)$. Thus we here proved the following.

Theorem 2.1: If $\mathscr{S}_{0}$ denotes the set of classes in $K_{0}(X)$ of the operators of the form $A \cup A^{*}$, then $\mathscr{S}_{0}=K_{0}(M ; Y)$.

In a similar way we consider the "odd case". Now $\operatorname{dim} M=2 k+1$ and it is well-known that in this case the Dirac operator $\partial_{X}: C^{\infty}(X ; S) \rightarrow C^{\infty}(X, S)$ is a generator of $K_{1}(\bar{X})$. Once again any class in $K_{1}(X)$ is the class of a differential operator. The following identity is the analogue of $(2.2)$ :

$$
\left[B_{1} \hat{\cup} B_{i 2}\right]=\left[\left(B_{1} \oplus B_{2}^{\prime}\right) \cup B_{2} \oplus\left(-B_{2}\right)\right]+\left[\left(-B_{2}\right) \cup B_{2}\right]
$$

Now we can deform the principal symbol of the operator $B_{2} \oplus\left(-B_{2}\right)$ to $I d$ through elliptic self-adjoint symbols and the operator $\left(B_{1} \oplus B_{2}\right) \cup\left(B_{2} \oplus\left(-B_{2}\right)\right)$ can be deformed to a self-adjoint operator of the form $(C \cup I d)$. Thus $K_{\mathrm{i}}(X)$ decomposes into $K_{1}(M)$ and the set $\mathscr{G}_{1}$ of classes of operators of the form $B \cup(-B)$. Thus, we have the following Theorem which covers the "odd case".

Theorem 2.2: If $G_{1}$ denotes the set of classes in $K_{1}(X)$ of the operators of the form $B \cup(-B)$, then $G_{1}=K_{1}(M, Y)$.

, Now we formulate our result in a different way. On $X$ we have the natural involution. $J$ which interchanges the two copies of $M$. The involution $J$ induces a map on the space of elliptic operators. 'We let $J_{0}$ denote the composition. of this map with taking adjoints. It is given by the formula $J_{0}(A)=J_{0}\left(A_{1} \cup A_{2}\right)=A_{2}^{*} \cup A_{1}{ }^{*}$. The corresponding map on the space of elliptic self-adjoint operators is given by the formula $J_{1}\left(B_{1} \cup B_{2}\right)=\left(-B_{2}\right) \cup\left(-B_{1}\right)$. Now we are able to formulate the results of this section in the following. way. 
Theorem 2.3: If $K_{i}^{J}(X)$ denotes the set of fixed points of the involution $J_{i}$, then $K_{i}(M, Y)=K_{i}{ }^{J}(X)$.

Remark: Recall that $\left[A^{*}\right]$ represents the inverse of $[A]$ in $K_{0}(X)$ and $[-B]$ the inverse of $[B]$ in $K_{1}(X)$. Thus $J_{i}$ modifies the operators to compensate for the change of orientation on the two halves of $X$.

\section{The pairing, of $K_{0}(M, Y)$ with $K^{0}(M, Y)$}

In this section we describe the intersection product

$$
K_{0}(M, Y) \times K^{0}(M, Y) \rightarrow \mathbb{Z}
$$

Thanks to our analytical representation we are able to apply here the results of the paper [12], in which the pairing for the case of a compact manifold without boundary is discussed. In this situation the intersection number is equal to the index of a suit= able operator. We begin by observing that the index of an operator of the form $A \cup A^{*}$ is equal to 0 .

Lemma 3:1: If $M$ is a compact manifold with boundary $Y$ and $A: C^{\infty}(M ; E)$ $\rightarrow C^{\infty}(M ; F)$ is a first order elliptic operator of the form (1.1) on the collar neighbouirhood of the boundary, then index $\left(A \cup A^{*}\right)=0$.

Proof: If $J: X \rightarrow X^{\prime}$ denotes the reflection on $X$, then we have $J\left(A \cup A^{*}\right) J^{\prime}$ $=A^{*} \cup A=\left(A \cup A^{*}\right)^{*}$. Hence index $\left(A \cup A^{*}\right)^{-}=0$

Therefore the pairing of $A \cup A^{*}$ with a class in $K^{0}(M ; Y)$ cannot be defined using the index of $A \cup A^{*}$. We must proceed differently to obtain a non-trivial index which yields the pairing of $A \cup A^{*}$ with an element of $K^{0}(M, Y)$. Fortunately we have the analytical representation of the pairing on the manifold without boundary (see [12]) and we have the commutative diagrams introduced in Section 2. These provide us with an elementary proof of the following proposition.

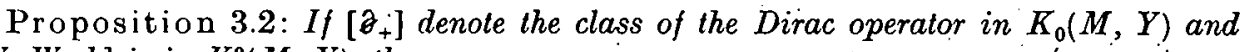
$[V, W ; h]$ is in $K^{0}(M, Y)$, then

$$
\left[\partial_{+}\right][V, W ; h]=\operatorname{index}\left(\partial_{+} \cup \partial_{+}^{*}\right) \otimes I d_{V \cup_{n} W} .
$$

More generally this formula works for any operator on $X$ realizing a class in $K_{0}(M, Y)$. That is, if $D$ is an elliptic operator such that $[D]$ is in $\mathscr{G}_{0}$, then the formula holds.

Remàr: It is not difficult to formulate a variant of Proposition 3.2 which includes the relative index formula of Cheeger-Gromov-Lawson (see [14)].

\section{The pairing of $K_{1}(M, Y)$ and $K^{1}(M, Y)$}

Now let us assume that $M$ is an odd dimensional spin ${ }^{c}$-manifold. In this case the I)irac operator $\partial_{M}: C^{\infty}(M ; S) \rightarrow C^{\infty}(M ; S)$ is a self-adjoint operator and its class is " generator of $K_{1}(M, Y)=K_{1}^{J}(X)$.

The pairing between $\mathrm{K}$-homology and $\mathrm{K}$-cohomology is now givèn by à spectral invariant, so we discuss the spectral properties of self-adjoint operators on closed manifolds for a while. Let $D: C^{\infty}(X ; V) \rightarrow C^{\infty}(X ; V)$ be a self-adjoint elliptic pseudodifferential operator of positive order on a closed manifold $X$. We know (see $[1,3]$ for 
instance). that $D$ has a discrete spectrum $\left\{\lambda_{k}\right\}_{k \in \mathbb{Z}}$. If we order the eigenvalues $\left|\lambda_{0}\right|$ $\leqq\left|\lambda_{1}\right| \leqq \cdots$, then there exists a constant $C>0$ and an exponent $\delta>0$ such that $\left|\lambda_{n}\right|>C n^{\delta}$ if $n>n_{0}$ is large. This implies that the eta-function $\eta(s, D)=\sum_{\lambda \neq 0} \operatorname{sign}(\lambda)$ $\times|\lambda|^{-8}$ is well-defined for $\operatorname{Re}(s) \gg 0$. Moreover it has a meromorphic extension to the whole complex plane and $s=0$ is a regular point. The absolute eta-invariant $\eta(0, D)$ is an important invariant of elliptic self-adjoint operators, which in some sense corresponds to the index. Here we present an analogue of Lemma 3.1 for the "odd case".

Proposition 4.1: Let $A: C^{\infty}(M ; E) \rightarrow C^{\infty}(M ; E)$ be a first order elliptic selfadjoint pseudodifferential operator having the form (1.1) on some collar neighbourhood of the boundary. If $G$ and $B$ do not depend on $t$ in the collar, $G$ is unitary, and $B$ is selfadjoint, then $\dot{\eta}(0, A \cup(-A))=0$.

Proof: It is obvious that $G^{2} \doteq-I d$ and $G B=-B G$ under the assumptions we have made. Now, let us consider the equation $(A \cup(-A)) u=\lambda u$ for $\lambda \neq 0$. A solution $\ddot{u}$ is of the form $u=u_{1}^{\prime} \cup u_{2}$, where $A u_{1}=\dot{\lambda} u_{1}, A u_{2}=-\hat{\lambda} u_{2}$ and $G u_{1}=u_{2}$ on some bicollared neighbourhood of $Y$ : If we define $\bar{u}=u_{2} \cup\left(-u_{1}\right)$, then $\bar{u}$ satisfies the equations $(A \cup(-A)) \tilde{u}=-\lambda \bar{u}$ and $G\left(\tilde{u}_{1}\right)=\tilde{u}_{2}$ on the same collar. This shows that $A \cup(-A)$ has a symmetric spectrum

Remarks: (i) In [20] it is shown that the operator $A \cup(-A)$ has trivial nullspace. (ii) Proposition 4.1 does not imply the vanishing of the local eta-invariant. The ete-function is given by the formula $\eta(s)=\Gamma((s+\hat{1}) / 2)^{-1} \int_{0}^{+\infty} t^{(s-1) / 2} \operatorname{tr}\left(D \mathrm{e}^{-t D^{2}}\right) d t$. The operator $D \mathrm{e}^{-t D^{2}}$ has a smooth kernel $K(t, x, y)$, and we define the local etafunction $\eta(s, x)=\Gamma((s+1) / 2)^{-1} \int_{0}^{+\infty} t^{(s-1) / 2} \operatorname{tr}(K(t, x, x)) d t$. It is well known (see [7]) that $\eta(s, x)$ is holomorphic for $\operatorname{Re}(s)>-2$ for generalized Dirac opérators. Although $\eta_{A \cup-A}(0)=0$, it is often the case that the local eta-invariant $\eta_{A U-A}(0, x)$ does not vanish. The application of this fact to the study of the eta-invariant on manifolds with boundary will be given elsewhere.

Now let us assume that $\left\{B_{\ell}\right\}_{t \in S^{1}}$ is a family of elliptic self-adjoint operators over $S^{1}$. The spectral flow sf $\left\{B_{\ell}\right\}$ of the family $\left\{B_{\ell}\right\}$ is given by the formula

$$
\operatorname{sf}\left\{B_{t}\right\}=\int_{0}^{1}\left(d \eta\left(B_{t}, 0\right) / d t\right) d t .
$$

It is just the difference between the number of the eigenvalues which change sign from - to + as the parameter changes from 0 to 1 and the number of eigenvalues which change sign from + to - (see $[2,9,19]$ ). It turns out that the pairing between $K_{1}$ and $K^{1}$ can be defined in terms of the spectral flow. The elements of $K^{1}(M, Y)$ are just the homotopy classes of the continuous maps $h: M \rightarrow \dot{U}(\infty)$ such that $h \mid Y=I d$. By a standard approximation argument we know that each class contains a smooth map $g: M \rightarrow U(N)$, which is equal to the identity on some collar of $Y$. Thus it extends to a map of the form $\tilde{g}=g \cup I d$ on the double of $M$.

We have two unitarily equivalent elliptic operators on $X$.

$$
B_{0}=.\left(\partial_{M} \cup-\partial_{M}\right) \otimes I d d_{\mathbf{C}^{N},} \quad B_{1}=(I d \otimes \tilde{g})((\hat{\theta} \cup-\hat{\theta}) \otimes I d)(I d \otimes \tilde{g})^{-1} .
$$

We form the family $\left\{B_{t}\right\}, B_{t}=t B_{1}+(1-t) B_{0}$, over $S^{1}$. The main result of this section is the following corollary from the corresponding result from [12]. 
Theorem 4.2: The formula $[\partial][h]=[(\partial \cup(-\partial)) \otimes I d][h \cup I d]=\operatorname{sf}\left\{B_{t}\right\}$ is true. More generally this formula holds for any operator $B$ realizing the class in $K_{1}{ }^{J}(X)$.

Remark: In [12] the intersection number is given as the index of a suitable Toeplitz operator. These realizations are equivalent and one can find a detailed discussion of this in $[9,19]$.

\section{Pairing with the image of the coboundary homomorphism}

In this section we discuss special cases of our results. The reason is that we want to obtain information about the contribution to the intersection numbers given by terms which depend only on the boundary data.

We start with the "even case". Let $M$ be an even-dimensional Spinc-manifold with boundary $Y$. We consider the exact sequence

$$
\begin{aligned}
& K^{\mathrm{c}}(M, Y) \rightarrow K^{\mathrm{c}}(M) \rightarrow K^{\mathrm{c}}(Y) \\
& \partial_{1} \uparrow \quad \downarrow^{\partial_{0}} \\
& K^{1}(Y) \leftarrow K^{1}(M) \leftarrow K^{1}(M, \dot{Y}) .
\end{aligned}
$$

Let us denote by $\gamma$ the element of $K^{1}(Y)$, which is defined by the map $g: Y \rightarrow U(N)$. Then using our notation we have.

$$
\begin{aligned}
& \dot{\partial}_{1} \gamma=\left[M \times \mathbb{C}^{N} \cup_{g} M \mathbb{C}^{N}\right]-\left[X \times \mathbb{C}^{N}\right]=\left[E^{g}\right]-\left[\mathbb{C}^{N}\right]
\end{aligned}
$$

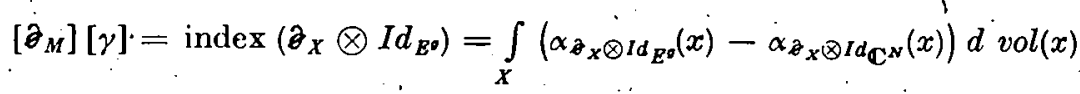

Here $\alpha_{A}$ denotes the index density of the operator $A$ (see [13: Thm 1.7.6]). The difference we have on the right side of our formula vanishes outside some collar of $Y$ and, in fact, gives the index density of an operator on $S^{1} \times Y$. One can find a detailed discussion of all this in $[9,19]$. Let us only mention here that we are able to express the above index in terms of spectral flow. If we fix a product metric on the collar, then $\hat{\partial}_{Y}$ is a Dirac operator on an odd-dimensional manifold which is self-adjoint. We put $B_{0}=\partial_{Y} \otimes I d_{\mathbb{C}^{N}}, B_{1}=(I d \otimes g)\left(\partial_{Y} \otimes I d\right)(I d \otimes g)^{-1}$ and define the family over $S^{\prime \prime}$ as in the previous section. We end up with the formula $\left[\partial_{M}\right] \partial_{1} \gamma=\operatorname{sf}\left\{B_{t}\right\}$.

The "odd case" is similar. If $M$ is an odd-dimensional Spinc-manifold, then $\partial_{M}$ decomposes as follows on the collar:

$$
\partial_{M}=G\left(\partial_{t}+\left(\begin{array}{ll}
0, & \partial_{-} \\
\partial_{+}, & 0
\end{array}\right)\right)
$$

where $\hat{\theta}_{+}: C^{\infty}\left(Y ; S^{+}\right) \rightarrow C^{\infty}\left(Y ; S^{-}\right)$is now the.Dirac operator on the even-dimensional manifold. Since $Y$ is a boundary, it follows that index $\hat{z}_{+}=0$ (see [17: Chap. XVII] or [6]). Now it is difficult to describe $\partial_{0}\left([E]-\left[\mathbb{C}^{r k E}\right]\right)$ directly. However, if we take the product of all manifolds with $S^{1}$ and use Bott periodicity, we end up with the formula $\left[\hat{\theta}_{M}\right] \partial_{0}\left([\dot{E}]-\left[\mathbb{C}^{r k E}\right]\right)=$ index $\left(\partial_{+} \otimes I d_{E}\right)$.

\section{REFERENCES}

[1] Atry $\mathrm{A}$, M. F., and R. Botт: The index problem for manifolds with boundary. In: Differential Analysis, Bombay Coll. 1964. London: Oxford Univ. Press 1964, p. 175-186.

[2] Atiyah, M. F., Patodr, V. K., and I. M. Straer: Spectral asymmetry and Riemannian geometry I, III. Math. Proc. Camb. Phil. Soc. 77 (1975), 43-69 and 79 (1976), 71-99. 
[3] Batm, P., and R. G. Dovaras: K-hiomology and index theory. Proc. Symp. Pure Math. (Amer. Math. Soc.) 38 (1982), 117-173.

[4] Badm, P., and R. G. Dodglas: Toeplitzoperators and Poincare duality. Proc. Toeplitz Mem. Conf., Tel Aviv 1981. Operator Theory, Adv. Appl. 4 (1982), 137-166.

[5] BA DM, P., and R. G. Dovglas: Analytic boundary cycles for K-homology. Preprint.

[6] BaUM, P., Dodglas, R. G., and M. E. TAYLOR: Cycles and relative cycles in analytic K-homology. J. Diff. Geometry (to appear).

[7] Bișut, J. M., and D. S. FreEd: The analysis of elliptic families: Dirac operators, eta invariant, and the holonomy theorem-of Witten. Comm. Math. Phys. 107 (1986); 103-163.

[8] BlaCKadAR, B.: K-theory for'operator algebras. New York: Springer-Verlag 1986.

[9] Booss, B., and K.P. WoJcIEchowskI: Desuspension of splitting elliptic symbols I, II. Ann. Glob. Analysis and Geometry 3 (1985); $337-383$ and 4 (1986), 349-400.

[10] Booss-BavnBek, B., and K.P. WoJcIEchowski: Elliptic boundary value problems for generalized Dirac operators (to appear).

[11] Connes, A.: Noncommutative differential geometry. Publ. Math. Inst. Hautes Etudes. Sci. $62(1986), 41-144$.

[12] Dodglas, R. G.: Invariant theory for elliptic operators. Proc. R. Ir. Acad. 86 A (1986), 161174.

[13] Grkmy, P. B.: Invariance Theory, the Heat Equation and the Atiyah-Singer Index Theorem (Math. Lect.'Ser. 11). Wilmington (Delaware, USA): Publish or Perish 1984.

[14ך Gromov, M., and B. Lawson:- Positive scalar curvature and the Dirac operator. Publ. Math. Inst. Hautes Etudes Sci. 58 (1983), 83-196.

[15] Hömander, L.: The Analysis of Linear Partial Differential Operators, Vol. 3. New York: Springer-Verlag 1985.

[16] Kasparov, G. G.: Topological invariants of elliptic operators. I: K-homology. Math. USSR-Izv. 9, 751-792.

[17] Palais, R. S: (ed.): Seminar on the Atiyah-Singer Index Theorem (Ann. Math. Studies 57). Princeton: Univ. Press 1965.

[18] Wojciecrowski, $\widehat{K}$. P.: Elliptic operators and relative K-homology groups on manifolds with boundary. C. R. Math. Acad.'Sci. Canada 7 (1985), $149-154$.

[19] Wojcíchowski, K. P.: Spectral flow and the general linear conjugation problem. Simon Stevin 59 (1985), $59-91$.

[20] WoJcrechowski, K. P.: On the Calderon projections and spectral projections of the elliptic operators. J. Operator Theory (to appear).

Manuskripteingang: 26. 09. 1988

\section{VERFASSFR:}

Prof. Dr. Ronald G. Dotglas College of Art and Sciences

State University of New York and Stony Brook

Stony Brook, NY 11794, USA
Dr. Krysztof P. Wojciechowski Institute of Mathematics University of Warsaw P-00-901 Warsaw 